\title{
Enhanced Shift Currents in Monolayer 2D GeS and SnS by Strain-Induced Band-Gap Engineering
}

Ngeywo Tolbert Kaner ${ }^{\mathrm{a}}$, Yadong Wei ${ }^{\mathrm{a}}$, Yingjie Jiang ${ }^{\mathrm{a}}$, Weiqi $\mathrm{Li}^{\mathrm{a} *}$, Xiaodong $\mathrm{Xu}^{\mathrm{a}}$, Kaijuan Pang ${ }^{\mathrm{a}}$, Xingji Li ${ }^{\mathrm{b}}$, Yang Jianqun ${ }^{\mathrm{b}}$, YongYuan Jiang ${ }^{\mathrm{a} *}$, Guiling Zhang ${ }^{\mathrm{c}}$, Weiquan $\operatorname{Tian}^{\mathrm{d} *}$

${ }^{\mathrm{a} S c h o o l ~ o f ~ P h y s i c s, ~ H a r b i n ~ I n s t i t u t e ~ o f ~ T e c h n o l o g y, ~ H a r b i n ~ 150001, ~ C h i n a ~}$

${ }^{b}$ Materials Science and Engineering, Harbin Institute of Technology, Harbin 150001, China

${ }^{\mathrm{c}}$ School of Materials Science and Engineering, Harbin University of Science and Technology, Harbin 150080, China

${ }^{\mathrm{d}}$ School of Chemistry and Chemical Enginnering, Chongqing University, Chongqing 401331, China

E-mail: tccliweiqi@ hit.edu.cn 
S2

SUPPLEMENTARY INFORMATION
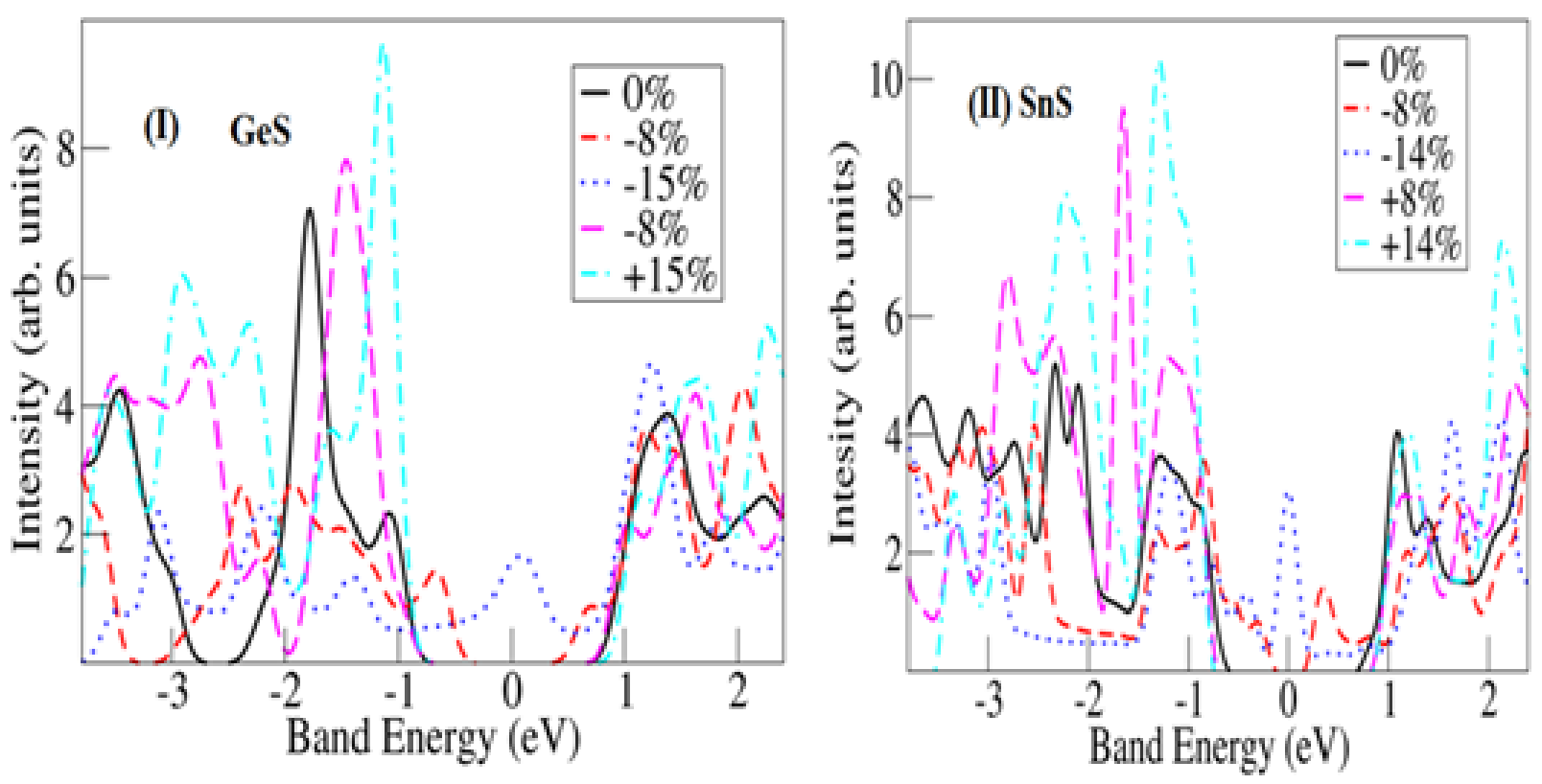

Figure S1: Evolution of the Density of States with Equibiaxial Strain Monolayer 2D GeS and SnS. The figure on the left is for Monolayer 2D GeS and on the right is for SnS. 

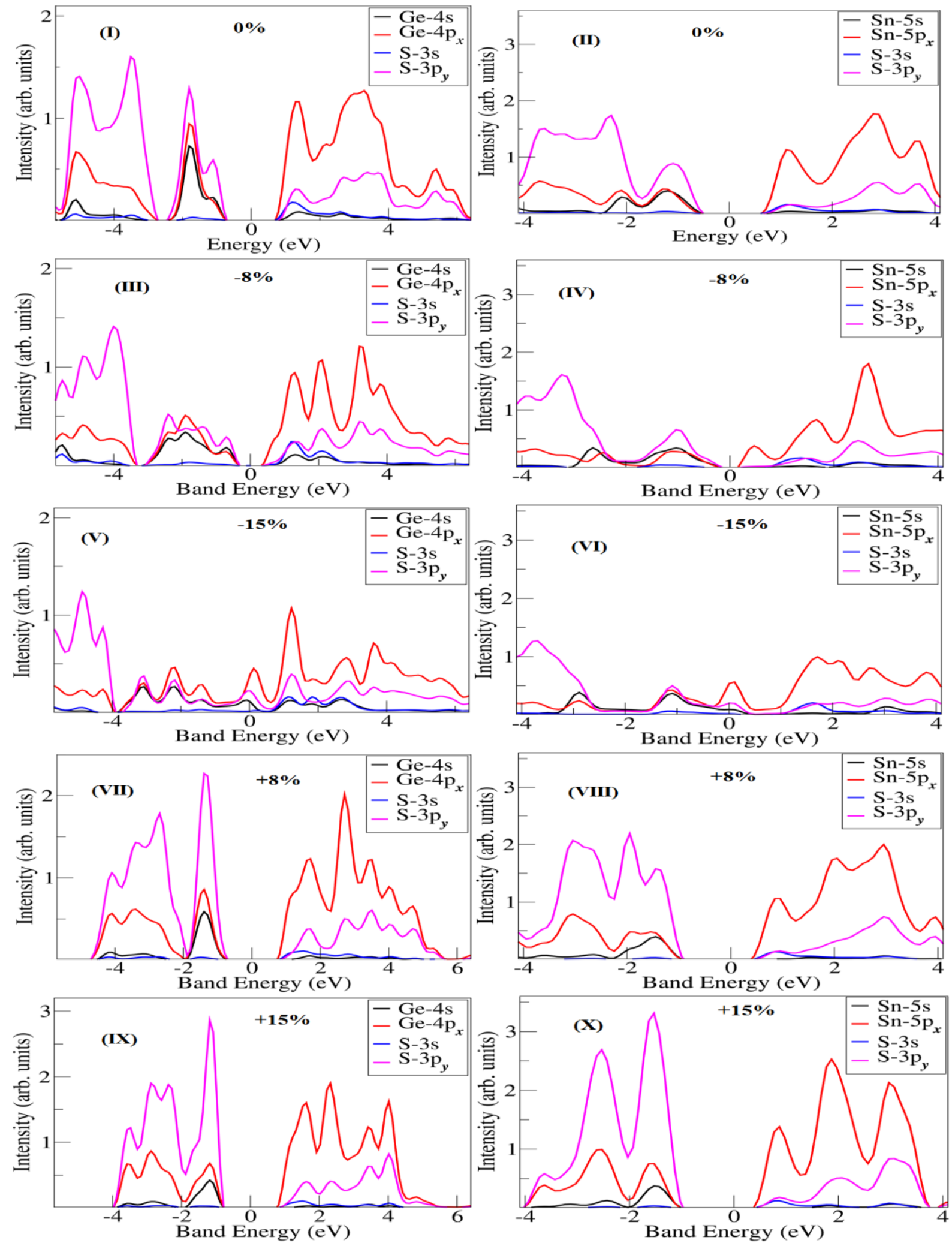

Figure S2: Evolution of the Projected Density of States with Equibiaxial Strain. Monolayer $2 D$ GeS and SnS. The left hand Column is for GeS while the right hand is column for SnS. 